\title{
Molecular clouds in galaxies as seen from NIR extinction studies
}

\author{
João Alves \\ Calar Alto Observatory, Almería, Spain \\ email: jalves@caha.es
}

\begin{abstract}
Near infrared dust extinction mapping is opening a new window on molecular cloud research. Applying a straightforward technique to near infrared large scale data of nearby molecular complexes one can easily construct density maps with dynamic ranges in column density covering, $3 \sigma \sim 0.5<\mathrm{A}_{V}<50$ mag or $10^{21}<N<10^{23} \mathrm{~cm}^{-2}$. These maps are unique in capturing the low column density distribution of gas in molecular cloud complexes, where most of the mass resides, and at the same time allow the identification of dense cores $\left(\mathrm{n} \sim 10^{4} \mathrm{~cm}^{-3}\right)$ which are the precursors of stars. For example, the application of this technique to the nearby Pipe Nebula complex revealed the presence of 159 dense cores (the largest sample of such object in one single complex) whose mass spectrum presents the first robust evidence for a departure from a single power-law. The form of this mass function is surprisingly similar in shape to the stellar IMF but scaled to a higher mass by a factor of about 3 . This suggests that the distribution of stellar birth masses (IMF) is the direct product of the dense core mass function and a uniform star formation efficiency of $30 \% \pm 10 \%$, and that the stellar IMF may already be fixed during or before the earliest stages of core evolution. We are now extending this technique to extragalactic mapping of Giant molecular Clouds (GMCs), and although a much less straightforward task, preliminary results indicate that the GMC mass spectrum in M83 and Centaurus A is a power-law characterized by $\alpha \sim-2$ unlike CO results which suggest $\alpha \sim-1$.
\end{abstract}

Keywords. ISM: structure, evolution, dust, individual (Pipe Nebula, M83), stars: mass function

\section{Introduction}

Little is understood about the internal structure of molecular clouds and consequently the initial conditions that give rise to star and planet formation. This is largely due to the fact that molecular clouds are primarily composed of molecular hydrogen, which is virtually inaccessible to direct observation. Therefore, the traditional methods used to derive the basic physical properties of such molecular clouds therefore make use of observations of trace $\mathrm{H}_{2}$ surrogates, namely those rare molecules with sufficient dipole moments to be easily detected by radio spectroscopic techniques, and interstellar dust, whose thermal emission can be detected by far infrared and radio continuum techniques (e.g., Motte et al. 1998, Lehtinen et al. 2001). However, the interpretation of results derived from these methods is not always straightforward (e.g., Alves, Lada, \& Lada 1999; Chandler \& Richer 2000). Several poorly constrained effects inherent in these techniques (e.g., deviations from local thermodynamic equilibrium, opacity variations, chemical evolution, small-scale structure, depletion of molecules, unknown emissivity properties of the dust, unknown dust temperature) make the construction of an unambiguous picture of the physical structure of these objects a very difficult task.

There is then a need for a less complicate and more robust tracer of $\mathrm{H}_{2}$ to access not only the physical structure of these objects but also to accurately calibrate molecular abundances and dust emissivity inside these clouds. The deployment of sensitive infrared array cameras on large telescopes, however, has fulfilled this need by enabling direct 


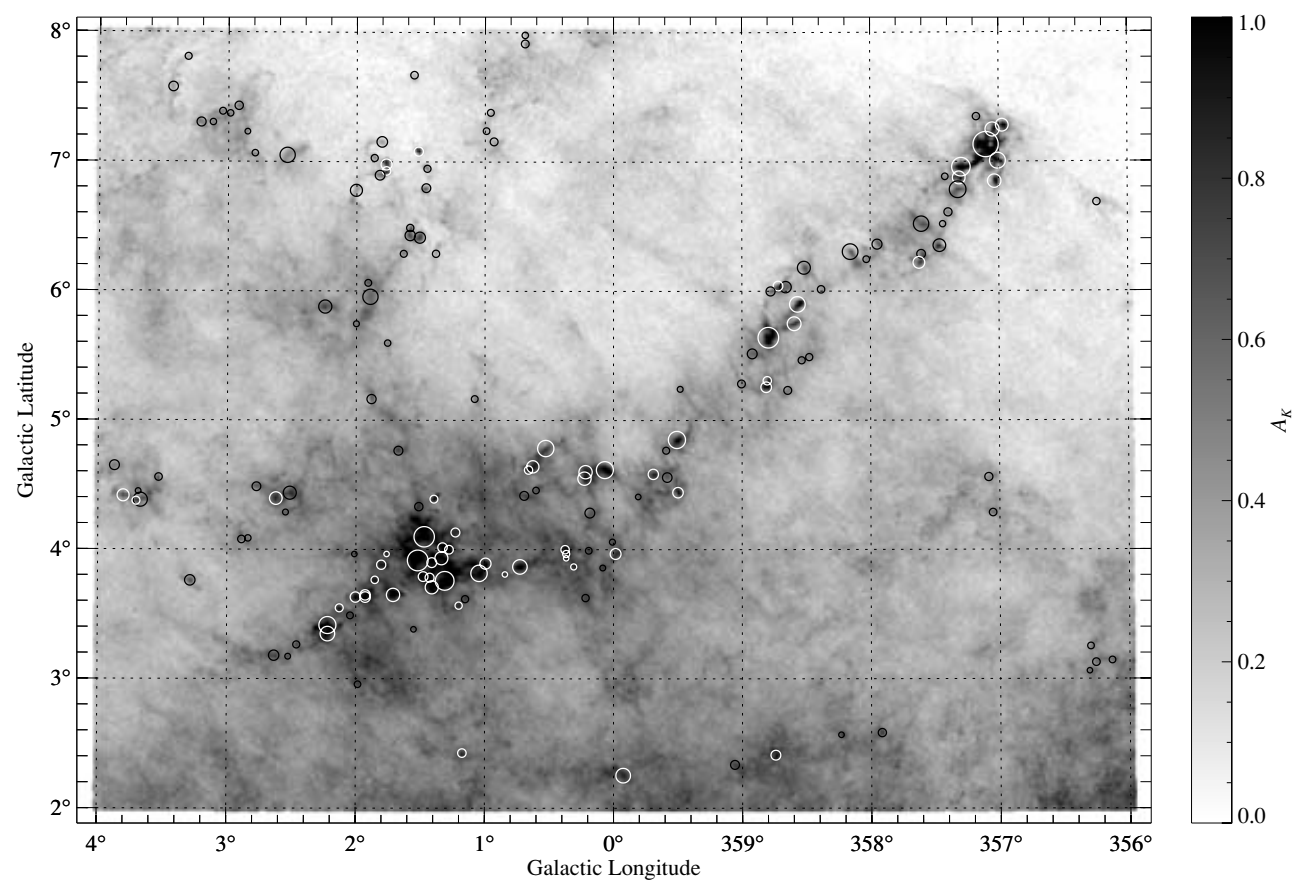

Figure 1. Dust extinction map of the Pipe nebula molecular complex from Lombardi, Alves, \& Lada (2006). This map was constructed from near-infrared observations of about 4 million stars in the background of the complex. Approximately 160 individual cores are identified within the cloud and are marked by an open circle proportional to the core radius. Most of these cores appear as distinct, well separated entities. Figure from Lombardi, Alves, \& Lada (2006)

and precise measurements of dust extinction toward thousands of individual background stars observed through a molecular cloud (Lada et al. 1994, Alves et al. 2001, Lombardi $\&$ Alves 2001). Such straightforward measurements are free from the complications that plague molecular-line or dust emission data and enable detailed maps of cloud structure to be constructed.

\section{Results from the Pipe Nebula}

In Figure 1 we present the $8^{\circ} \times 6^{\circ}$ extinction map of the Pipe Nebula complex constructed with 4 million stars from the 2MASS database (from Lombardi, Alves, Lada 2006). This molecular complex is one of the closest to Earth $(\sim 130 \mathrm{pc})$ of this size and mass, and it is particularly well positioned along a relatively clean line of sight to the rich star field of the Galactic bulge, which given the close distance of the Pipe nebula allowed us to achieve spatial resolutions of $\sim 0.03 \mathrm{pc}$, or about 3 times smaller the typical dense core size. The complex also exhibits very low levels of star formation suggesting that its dense cores likely represent a fair sample of the initial conditions of star formation. We present in Figure 2 the probability density function for the 159 core masses identified in the Pipe Nebula complex (marked in Figure 1). The core mass distribution seems to be characterized by two power-laws and a well defined break point at $\sim 2 \mathrm{M}_{\odot}$. For comparison we also present the field star IMF determined by Kroupa (2001) (solid grey) and Chabrier (2003) IMF (dotted grey), and the IMF for the young Trapezium cluster (dashed grey) (Muench et al. 2002). All these stellar IMFs were shifted to higher masses by an average scale factor of about 3 . The point of this comparison is to simply 


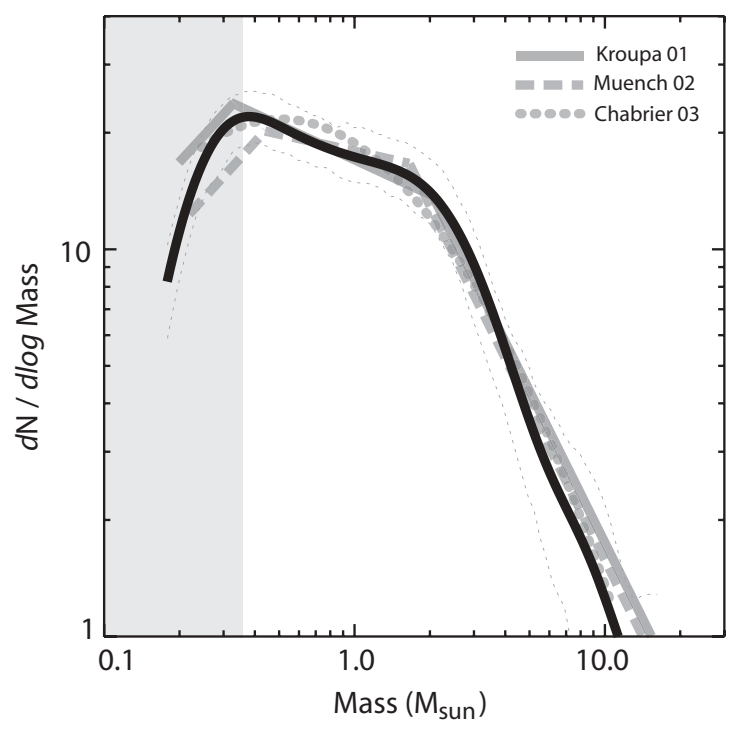

Figure 2. Probability density function of Pipe Nebula core masses (black). The grey region indicates sample incompleteness while the two thin dashed line indicates the $90 \%$ confidence limits. The core mass distribution seems to be characterized by two power-laws and a well defined break point at around $2 \mathrm{M}_{\odot}$. Also plotted are the field star IMFs of Kroupa (2001) (solid grey) and Chabrier (2003) (dotted grey), and the Muench et al. (2002) (dashed grey) IMF all scaled up by a factor of $\sim 3$ in mass. Figure from Alves, Lombardi, and Lada (2007).

illustrate the overall similarity between different stellar IMFs from different environments and constructed in different ways, and the dense core mass function of the Pipe Nebula. Considering the overall uncertainties, apparent differences between the different distributions are likely not significant.

\section{Conclusions and Future Work}

The close similarity in shape between the dense core mass function and the IMF supports a general concept of a 1-to-1 correspondence between the individual dense cores and soon to be formed stars. In this respect our observations are consistent with and appear to confirm the results of dust continuum surveys in other clouds (e.g., Motte et al. 1998). However, our study provides the first robust evidence for a departure from a single power law in the core mass function. We find that the location of the break indicates that there is a factor of about 3 difference in mass scale between the two distributions, which implies that a uniform SFE, that we estimate to be $30 \% \pm 10 \%$, will likely characterize the star formation in these dense cores, across the entire span of (stellar) mass.

We are currently expanding the technique to map Giant Molecular Clouds (GMCs) in nearby galaxies via seeing or diffraction limited NIR imaging. These clouds can be mapped in a manner analogous to the extinction map in figure 1 and with sub-arcsec resolution (corresponding to few to tens of parsecs in nearby galaxies), although the interpretation of the observed reddening and light attenuation into physical quantities is hampered by effects such as the unknown geometry of the system and non-negligible scattering of NIR light by dust particles. A first investigation on the importance of these effects can be found in Kainulainen, Juvela, and Alves (2007). Preliminary results 


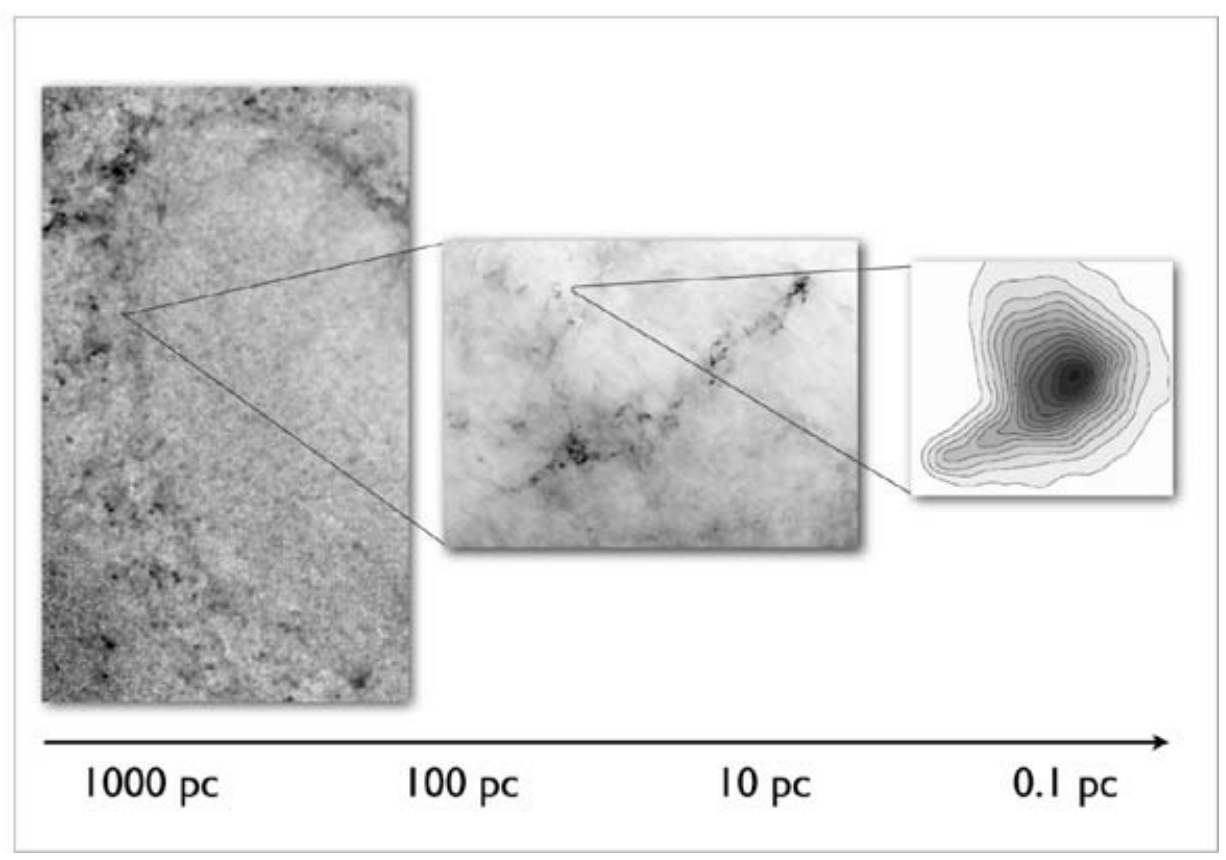

Figure 3. High signal-to-noise column density maps across 4 orders of magnitude in size, using one single tracer: extinction by dust grains. From the large to the small: M83, Pipe Nebula, and Barnard 68. Near infrared extinction mapping is opening a new window on molecular cloud research and is very complementary to traditional line and dust emission techniques.

indicate that the GMC mass spectrum in M83 and Centaurus A (Bialetski et al. 2007) is a power-law characterized by $\alpha \sim-2$ unlike $\mathrm{CO}$ results which suggest $\alpha \sim-1$.

\section{References}

Alves, J., Lada, C. \& Lada, E. 1999, ApJ 515, 265

Alves, J., Lada, C. \& Lada, E. 2001, Nature 409, 159

Alves, J., Lombardi, M. \& Lada, C. 2007, $A \& A$ in press

Bialetski, Y., Alves, J. et al. 2007, A\&A in preparation

Chabrier, G. 2003, ApJ 586, 133

Chandler, C. \& Richer, J. 2000, ApJ 530, 851

Kainulainen, J., Juvela, M. \& Alves, J. 2007, A\&A in press

Kroupa, P. 2001, MNRAS, 322, 231

Lada, C. J., Lada, E. A., Clemens, D. P. \& Bally, J. 1994, ApJ 429, 694

Lombardi, M. \& Alves, J. 2001, A\& A 377, 1023

Lombardi, M., Alves, J. \& Lada, C. 2006, A\&A 454, 781

Lehtinen, K., Haikala, L. K., Mattila, K. \& Lemke, D. 2001, A $\& A$ 367, 311

Muench, A. A., Lada, E. A., Lada, C. J. \& Alves, J. 2002, ApJ 573, 366

Motte, F., Andre, P. \& Neri, R. 1998, A\&A 336, 150 